\title{
Article
}

\section{Talking About My Generation: the Date of the West Kennet Long Barrow}

\author{
Wysocki, Michael Peter, Wittle, A and Bayliss, A \\ Available at http://clok.uclan.ac.uk/10754/ \\ Wysocki, Michael Peter, Wittle, A and Bayliss, A (2007) Talking About My \\ Generation: the Date of the West Kennet Long Barrow. Cambridge \\ Archaeological Journal, 17 (S1). pp. 85-101.
}

It is advisable to refer to the publisher's version if you intend to cite from the work. http://dx.doi.org/10.1017/S0959774307000182

For more information about UCLan's research in this area go to

http://www.uclan.ac.uk/researchgroups/ and search for <name of research Group>.

For information about Research generally at UCLan please go to http://www.uclan.ac.uk/research/

All outputs in CLoK are protected by Intellectual Property Rights law, including Copyright law. Copyright, IPR and Moral Rights for the works on this site are retained by the individual authors and/or other copyright owners. Terms and conditions for use of this material are defined in the policies page.

\section{CLoK}

Central Lancashire online Knowledge www.clok.uclan.ac.uk

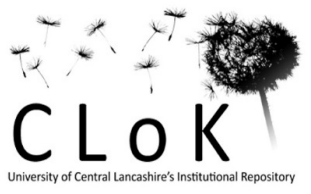




\title{
Talking About My Generation: the Date of the West Kennet Long Barrow
}

\author{
Alex Bayliss, Alasdair Whittle \& Michael Wysocki
}

Thirty-one radiocarbon results are now available from the West Kennet long barrow, and are presented within an interpretive Bayesian statistical framework. Two alternative archaeological interpretations of the sequence are given, each with a separate Bayesian model. In our preferred interpretation, the barrow is seen as a unitary construction (given the lack of dating samples from the old ground surface, ditches or constructional features themselves), with a series of deposits of human remains made in the chambers following construction. Primary deposition in the chambers is followed by further secondary deposition of some human remains, including children, and layers of earth and chalk, the latest identifiable finds in which are Beaker sherds. In the Bayesian model for this sequence, the construction of the monument at West Kennet, as dated from the primary mortuary deposits, occurred in 3670-3635 cal. BC, probably in the middle decades of the thirty-seventh century cal. BC. The last interments of this initial use of the chambers probably occurred in 3640-3610 cal. вс. The difference between these two distributions suggests that this primary mortuary activity probably continued for only 10-30 years. After a hiatus probably lasting for rather more than a century, the infilling of the chambers began in 3620-3240 cal. ВС and continued into the second half of the third millennium cal. BC. In an alternative interpretation, we do not assume that all the people dated from the primary mortuary deposits were placed in the monument in a fleshed or partially articulated condition; they could therefore have died before the monument was built, although they must have died before the end of the formation of the mortuary deposit. In the Bayesian model for this interpretation, the monument appears to belong either to the thirty-seventh century cal. BC or the mid-thirtysixth century cal. $B C$, and deposition again appears short-lived, but the model is unstable.

Results are discussed in relation to the setting and sequence of the local region.

The West Kennet long barrow is one of the best known prehistoric field monuments in Britain. It was recognized from at least the time of John Aubrey in the seventeenth century, and was partially excavated by John Thurnam in the nineteenth century and more extensively by Stuart Piggott and Richard Atkinson in the 1950s (Thurnam 1860; Piggott 1962). Positioned just above the upper Kennet valley in the region around Avebury, north Wiltshire (SU 1046 6774; 5124'31" $\left.\mathrm{N}, 01^{\circ} 51^{\prime} 03^{\prime \prime} \mathrm{W}\right)$, it is the longest barrow in the local group. The barrow is flanked by a ditch on each side, and contains transepted orthostatic chambers at its eastern end. These consist of an end chamber, two pairs of opposed chambers, and a passage, principally formed by sarsen orthostats (Fig. 1). The passage led in from what was originally a concave orthostatic forecourt, which was later infilled and blocked by a façade of monumental sarsen uprights (Piggott 1962). Only a little of the mound behind the chambers was investigated, revealing a core of small sarsen stones piled on a buried soil, and capped by chalk rubble (Piggott 1962, fig. 3); the existence of a turfline above 




Figure 1. The West Kennet long barrow.

this sarsen core could suggest that this feature was part of an earlier, perhaps free-standing version of the monument. Likewise, a kink in the flanking ditches about a third of the way from the western end, identified by the original 1955 resistivity survey, has suggested that the mound may be of more than one phase (Richard Bradley pers. comm.; Martyn Barber pers. comm.; see also North 1996, fig. 26; Gibson 2000). Neither possibility has been tested by excavation since the 1950s; more recent geophysical survey by English Heritage has in fact failed to find the ditch kink (Mar- tin 2001). Investigation of land snails from a small part of the buried soil, well west of the façade, was carried out by John Evans at the end of the 1960s (Evans 1972, 263-4).

\section{Primary deposits}

The monument is thus notable as an imposing chambered long barrow, generally thought of as part of the wider Cotswold transepted type, but it is also remarkable for the contents of its chambers. Thurnam's investigation came down through the end chamber, revealing inhumations, and went some way along the passage, encountering substantial secondary backfill. Piggott and Atkinson's excavations showed similar backfill in the side chambers, covering further primary inhumations. These primary mortuary deposits consisted of skeletal remains of people of all ages and both sexes, in varying states of articulation and completeness, as well as a small cremation deposit. It was originally estimated that at least 43 individuals were represented in the primary deposits (Piggott 1962, 24; but see Wells 1962, 80). It was later suggested that these remains could be seen as arranged by some sort of categorization on the basis of age and sex: males in the end chamber, predominantly adults (men and women) in the inner side chambers, and juvenile and old people in the outer side chambers (Thomas \& Whittle 1986, 133). Subsequent re-analysis has shown, however, that Piggott's original estimate of numbers was too high; a better estimate is given in Figure 2, suggesting that 36 individuals are represented in the primary deposits (Wysocki \& Whittle in prep.).

Piggott and Wells argued that burial in the chambers, following interment or exposure elsewhere, was unlikely (Piggott 1962, 67), and seemed to have envisaged a process of successive deposition of fleshed or otherwise articulated bodies, with the subsequent removal of certain bones, notably skulls and long bones (Piggott 1962, 68). Incomplete disarticulated and commingled remains were therefore explained by a 
Figure 2. Revised demographic distribution for the primary deposits.

\begin{tabular}{|c|c|c|}
\hline & $\begin{array}{l}\text { W Chamber } \\
4 \text { o : 17-25 yrs } \\
\text { (1 Child) }\end{array}$ & \\
\hline $\begin{array}{c}\text { SW Chamber } \\
5 \text { Adult } \delta^{\top}: 20-50 \text { yrs } \\
1 \text { Subadult ơ: } 16-21 \text { yrs } \\
6 \text { Adult }+ \text { : } 20-40 \text { yrs } \\
1 \text { Subadult: } 12-15 \text { yrs }\end{array}$ & & $\begin{array}{c}\text { NW Chamber } \\
3 \text { Adult ơ: } 25-45 \text { yrs } \\
3 \text { Adult }+: 25-45 \text { yrs } \\
1 \text { Adult: } 25-45 \text { yrs } \\
\text { 1 Child: } 3-4 \text { yrs (mandible } \\
\text { and cranial fragment only) }\end{array}$ \\
\hline $\begin{array}{c}\text { SE Chamber } \\
1 \text { Adult ơ: 25-35 yrs } \\
1 \text { Adult }+ \text { : } 25-35 \text { yrs } \\
5 \text { Children: 3-6 yrs }\end{array}$ & & $\begin{array}{l}\text { NE Chamber } \\
1 \text { Adult o: } 30-40 \text { yrs } \\
1 \text { Adult ㅇ: } 20-25 \text { yrs } \\
1 \text { Adult ?+: ?35+ yrs }\end{array}$ \\
\hline
\end{tabular}

combination of successive interment and subsequent selective abstraction.

Both the style of the monument and artefacts associated with the primary interments suggested a date in the earlier part of the Neolithic (Piggott 1962, 71). That date was further supported with three radiocarbon dates then recently obtained from Windmill Hill causewayed enclosure nearby (Piggott 1962, 72; cf. Smith 1965).

\section{Secondary deposits}

Following the active mortuary use of the tomb and the partial collapse of sections of internal dry stone walling, the contents were sealed beneath deliberately introduced deposits of chalk rubble, earth and sarsen lumps which filled the chambers and passage to the roof. This secondary fill, excavated by Piggott and Atkinson from the side chambers and a portion of the passage untouched by Thurnam, contained pottery sherds ranging in style from Ebbsfleet Ware to Wessex-Middle Rhine Beaker together with many other artefacts (leaf-shaped arrowheads, flint scrapers and knives, whetstones, beads, bone scoops, pins and boar's tusks, to name a few). It was Piggott's considered view that the backfill represented material and artefacts accumulated over a very long period of time elsewhere, to be finally deposited as a ceremonial blocking of the monument, in a single act, at a time given by the latest ceramic style, Beaker (Piggott 1962, 68-71). Piggott argued that West Kennet could have been in use as a mortuary facility for several hundred years before being sealed off. The notion that megalithic chambered tombs remained open for use over a span of centuries became widely accepted. This view was later challenged, first for West Kennet, when Thomas \& Whittle (1986) suggested that the secondary fill was more probably a much more gradual process of deposition over a long period of time, and then by Saville (1990) on the basis of radiocarbon dates from Hazleton.

It can now be shown that there was also considerably more adult and immature human material deposited in among the secondary backfill than intimated in Piggott's original report (see below). These human remains were dispersed throughout the secondary fill in loose groups or sub-assemblages and have been crucial to this dating project.

\section{Objectives of this study}

Further dating of the West Kennet long barrow was undertaken principally because of the methodological advances in radiocarbon dating and the interpretation of radiocarbon dates which have been made in the last 


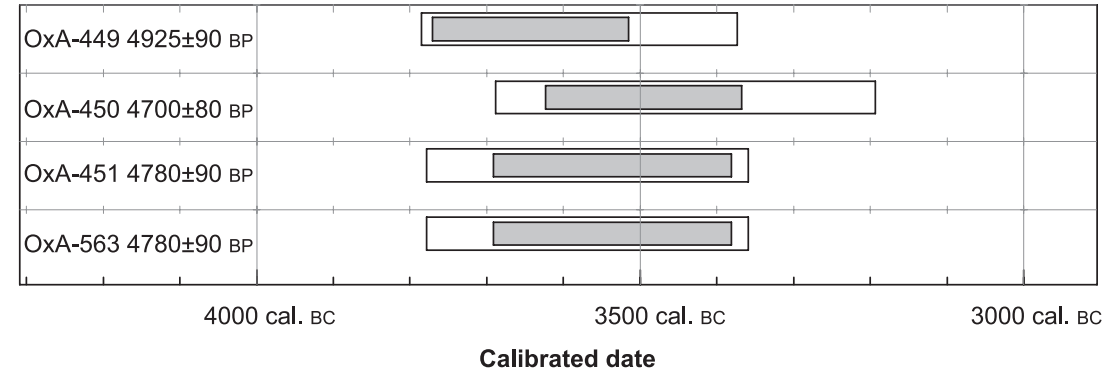

Figure 3. Radiocarbon dates for the long barrow obtained in 1984, calibrated using the maximum intercept method (Stuiver \& Reimer 1986) and data from Pearson et al. (1986). Shaded bars represent 68\% confidence intervals; white bars indicate $95 \%$ confidence intervals.

decade or so (Bayliss et al. this issue). These developments provide the potential to produce much more precise dating for such monuments (Bayliss \& Bronk Ramsey 2004; Bayliss et al. 1997). A more detailed understanding of the chronology of the Neolithic monuments within the Avebury area has also recently been identified as a research priority in the World Heritage Site (AAHRG 2001), one which would also contribute to public understanding and appreciation of this significant monument.

Specifically, the new dating programme was designed to address the following objectives:

- to date the primary construction of the monument and, if appropriate, the sequence of construction;

- to determine the dates of the mortuary deposits and their chronological span;

- to determine whether there was spatial variation in the deposition of human remains within a burial chamber over time;

- to determine whether the mortuary deposits in the different chambers were of different dates;

- to determine whether the articulated burials in the primary deposits were of a different date from the disarticulated material;

- to clarify the chronology of the secondary filling of the monument and to establish the date of final closure;

- to establish the relative position of West Kennet in the typological sequence of long barrows (Corcoran 1969; Darvill 1982; Saville 1990).

Such work at West Kennet was also timely as the detailed osteological review undertaken by Wysocki and Whittle as part a wider project on human remains and mortuary processes enabled the strategic selection of samples from specific individuals and contexts. In the course of the osteological work on West Kennet, it soon became apparent that there were significant differences between the original report and the sur- viving skeletal archive, and that the published account required revision, including of its failure to distinguish in some cases between material from primary and secondary contexts. Full details will be provided elsewhere (Wysocki \& Whittle in prep.). This recent study also suggests that there are no direct taphonomic traces evident to implicate prior exposure or burial elsewhere for any of the material from the primary deposits. Indeed, a number of individuals were clearly deposited in an articulated state as can be seen from the original plans (Piggott 1962, fig. 8). The patterns of disarticulation seen in partially articulated individuals do not conform to expected sequences of ligament decomposition (e.g. Haglund 1997a,b; Roksandić 2002) and rather suggest later disturbance of deposited skeletonized corpses.

\section{Sampling}

A simulation of the likely chronology of the monument was constructed to assess the number of samples which would be required to answer these questions to a resolution which would be archaeologically useful (Fig. 4). This was done using the R_Simulate function of OxCal (version 3.5) with the calibration curve of Stuiver et al. (1998), archaeological estimates for the likely date of the material (see Fig. 3), and estimated error terms for the radiocarbon measurements based on the available samples.

Certain types of sample were targeted for dating. In particular, samples which could not be from a secondary context were preferred. The categories of material selected for dating from West Kennet were:

- articulated bone groups which could not have been deposited more than a few years or so after the death of the individual concerned without becoming disarticulated (Mant 1987, 71; Roksandić 2002);

- bone groups where articulated deposition could be inferred because of the proportion of the skeleton identified in the archive;

- disarticulated human remains from individuals who are identifiably distinct on the basis of osteological duplications.

All specimens were selected to ensure that each dated sample was from a separate individual. This allows measurements on the same body to be combined before calibration, so that all dates included in the 
models are statistically independent (Bronk Ramsey 2001, 357). In addition, sampling locations on individual bones were chosen to avoid any areas showing previous use of consolidant or adhesives.

The first series of samples submitted from West Kennet, in December 2000, was selected to clarify the sequence of construction, use and disuse of the monument. Once these results were received, and a preliminary model constructed, further samples were selected to resolve problems raised by the first set of results and address more detailed objectives. In this case, samples of disarticulated human bone from the primary mortuary deposits were selected to see whether they were of different date from the articulating material from the same contexts.

Unfortunately, shortly after the second series of measurements had been completed, a technical problem was identified with the bone preparation method used in the Oxford Laboratory (Bronk Ramsey et al. 2004a; Bayliss et al. this issue). The resolution of this problem necessitated a third series of replicate samples.

\section{Results}

Thirty-one radiocarbon results are now available from West Kennet (Table 1). They come from 25 different human skeletons and one goat skeleton. Nineteen of the dated individuals come from the primary mortuary deposits. The other six human samples and the goat come from the secondary deposits.

The results are conventional radiocarbon ages (Stuiver \& Polach 1977). The calibrated date ranges provided in Table 1 have been calculated using the maximum intercept method (Stuiver \& Reimer 1986); all other distributions are based on the probability method (Stuiver \& Reimer 1993). All results have been calibrated using OxCal (v3.10) (Bronk Ramsey 1995; 1998; 2001) and data from Reimer et al. (2004).

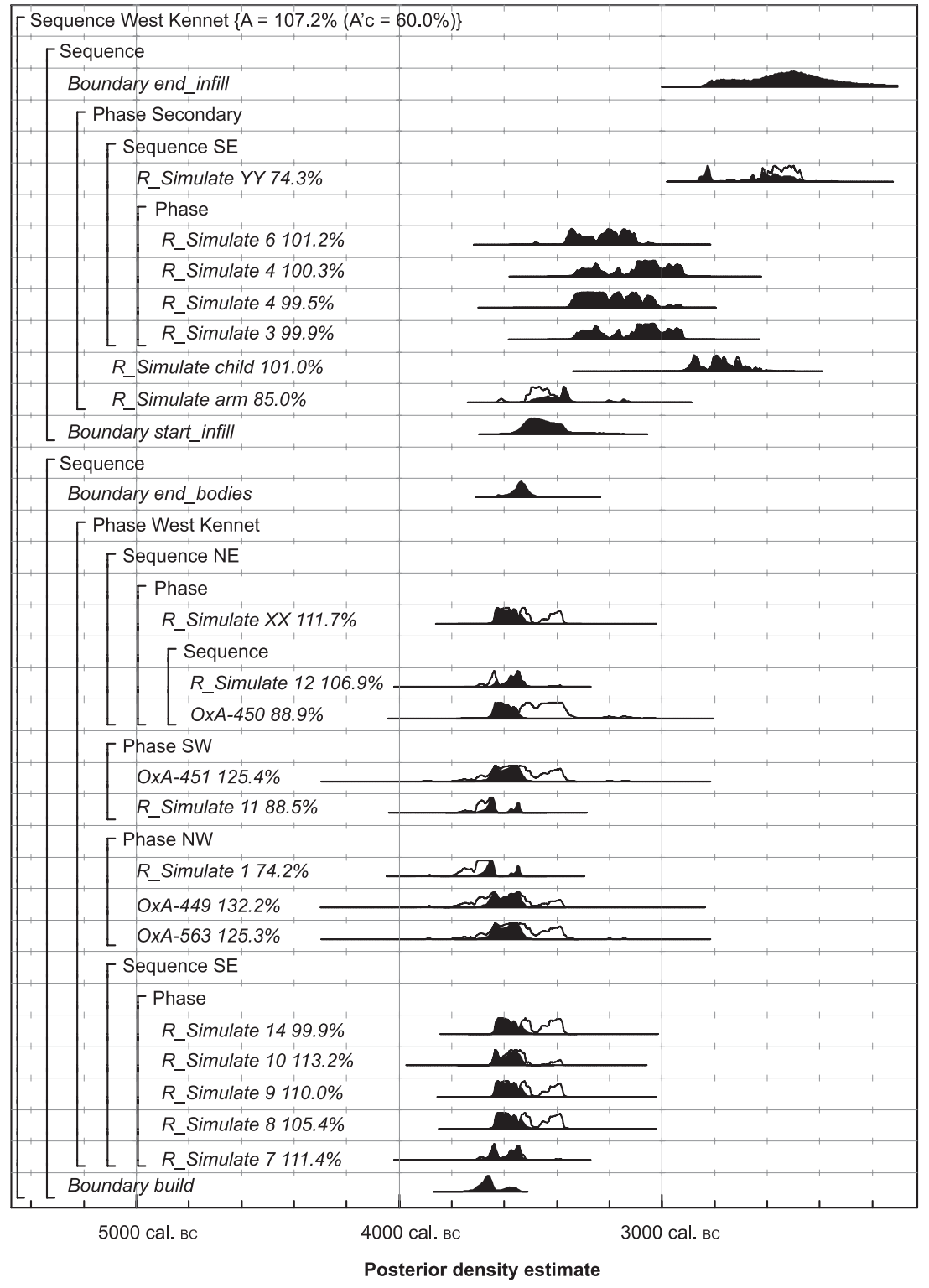

Figure 4. Probability distributions of simulated dates from West Kennet. Each distribution represents the relative probability that an event occurs at a particular time. For each radiocarbon date, two distributions have been plotted: one in outline which is the result of simple radiocarbon calibration, and a solid one based on the chronological model used; the 'event' associated with, for example, YY, is the growth of the person whose bones were dated. The other distributions correspond to aspects of the model. For example, the distribution 'build' is the posterior density estimate for the first burial activity on this site. The large square brackets down the left-hand side and the OxCal keywords define the overall model exactly.

The first four samples from the West Kennet long barrow were dated by the Oxford Radiocarbon Accelerator Unit in 1984 (OxA-449-OxA-451 and OxA536; Gillespie et al. 1985; Gowlett et al. 1986a,b). These samples were processed and measured according to 
Alex Bayliss et al.

Table 1. Radiocarbon measurements from the West Kennet long barrow. Results denoted by * have been undertaken on re-purified gelatin (see Bayliss et al. this issue).

\begin{tabular}{|c|c|c|c|c|c|c|c|c|}
\hline $\begin{array}{l}\text { Laboratory } \\
\text { no. }\end{array}$ & Sample no. and material & $\begin{array}{l}\text { Radiocarbon } \\
\text { age (BP) }\end{array}$ & $\delta^{13} \mathrm{C}(\%)$ & $\begin{array}{c}\delta^{15} N \\
(\%)\end{array}$ & $\begin{array}{l}\mathrm{C}: \mathrm{N} \\
\text { ratio }\end{array}$ & $\begin{array}{l}\text { Weighted } \\
\text { mean (BP) }\end{array}$ & $\begin{array}{l}\text { Calibrated date } \\
\text { range }(95 \% \\
\text { confidence) }\end{array}$ & $\begin{array}{l}\text { Posterior density } \\
\text { estimate (probability) }\end{array}$ \\
\hline GrA-23178 & \multirow[b]{2}{*}{$\begin{array}{l}\text { WK 2, left femur from child, c. 3-4 years, } \\
\text { from SE chamber primary deposit } \\
\text { This skeleton was disarticulated but } \\
\text { sufficient material has been recorded to } \\
\text { strongly suggest that it was articulated } \\
\text { when deposited. }\end{array}$} & $4835 \pm 45$ & -21.6 & 10.7 & & \multirow{2}{*}{$\begin{array}{l}4802 \pm 29 \\
T^{\prime}=0.9 \\
T^{\prime}(5 \%)=3.8 \\
v=1\end{array}$} & \multirow[t]{2}{*}{$3650-3520$ cal. вс } & \multirow{2}{*}{$\begin{array}{l}3650-3620 \mathrm{cal} . \text { BC }(79 \%) \text { or } \\
3565-3530 \mathrm{cal} . \text { BC }(16 \%)\end{array}$} \\
\hline OxA-13179* & & $4778 \pm 38$ & -20.8 & 11.0 & 3.3 & & & \\
\hline GrA-23179 & \multirow[b]{2}{*}{$\begin{array}{l}\text { WK 4, left femur from child, } c \text {. } \\
4-5 \text { year, from SE chamber primary } \\
\text { deposit } \\
\text { This skeleton was disarticulated but } \\
\text { sufficient material has been recorded } \\
\text { to strongly suggest that it was } \\
\text { articulated when deposited. }\end{array}$} & $4855 \pm 45$ & -21.4 & 10.1 & & \multirow{2}{*}{$\begin{array}{l}4818 \pm 30 \\
T^{\prime}=1.2 \\
T^{\prime}(5 \%)=3.8 \\
v=1\end{array}$} & \multirow[t]{2}{*}{ 3660-3520 cal. вс } & \multirow{2}{*}{$\begin{array}{l}3655-3620 \mathrm{cal} . \text { вС }(80 \%) \text { or } \\
3560-3535 \mathrm{cal} . \text { ВС }(15 \%)\end{array}$} \\
\hline OxA-13180* & & $4787 \pm 41$ & -21.1 & 8.8 & 3.3 & & & \\
\hline OxA-13241* & $\begin{array}{l}\text { WK 3, left femur from child, c. 3.5-4.5 } \\
\text { years, from SE chamber primary } \\
\text { deposit } \\
\text { This skeleton was disarticulated but } \\
\text { sufficient material has been recorded } \\
\text { to strongly suggest that it was } \\
\text { articulated when deposited. }\end{array}$ & $4806 \pm 36$ & -21.7 & 9.5 & 3.1 & & 3660-3520 cal. вс & $\begin{array}{l}3655-3620 \mathrm{cal} . \text { BC }(79 \%) \text { or } \\
3565-3530 \mathrm{cal} . \text { BC }(16 \%)\end{array}$ \\
\hline OxA-13200* & $\begin{array}{l}\text { WK 14, right femur from skeleton SE } \\
\text { X, adult female, SE chamber primary } \\
\text { deposit } \\
\text { Recovered disarticulated, although much } \\
\text { of the skeleton is present suggesting that } \\
\text { the individual was at least partially } \\
\text { articulated on deposition. }\end{array}$ & $4872 \pm 38$ & -20.6 & 9.4 & 3.1 & & 3710-3540 cal. вс & $\begin{array}{l}3655-3625 \mathrm{cal} . \text { Bс }(81 \%) \text { or } \\
3560-3535 \mathrm{cal} . \text { ВС }(14 \%)\end{array}$ \\
\hline OxA-13199* & $\begin{array}{l}\text { WK 13, right femur from skeleton SE } \\
\text { IX, adult male, SE chamber primary } \\
\text { deposit } \\
\text { Recovered disarticulated, although much } \\
\text { of the skeleton is present, suggesting that } \\
\text { the individual was at least partially } \\
\text { articulated on deposition. }\end{array}$ & $4880 \pm 38$ & -20.4 & 10.6 & 3.1 & & 3710-3540 cal. вс & $\begin{array}{l}3660-3625 \mathrm{cal} . \text { BC }(81 \%) \text { or } \\
3555-3535 \mathrm{cal} . \text { BC }(14 \%)\end{array}$ \\
\hline OxA-12653 & $\begin{array}{l}\text { WK } 16 \text {, right scapula, partially } \\
\text { articulated skeleton NW I, adult male, } \\
\text { NW chamber primary deposit }\end{array}$ & $4803 \pm 32$ & -19.6 & 11.8 & 3.3 & $\begin{array}{l}4847 \pm 27 \\
T^{\prime}=6.2 \\
T^{\prime}(5 \%)=3.8 \\
v=1\end{array}$ & 3670-3540 cal. вс & $\begin{array}{l}3655-3625 \mathrm{cal} . \text { BC }(81 \%) \text { or } \\
3555-3535 \mathrm{cal} . \text { BC }(14 \%)\end{array}$ \\
\hline GrA-23181 & & $4950 \pm 50$ & -20.9 & & & & & \\
\hline OxA-563 & $\begin{array}{l}\text { replicate of OxA-12653 and GrA- } \\
\text { 23181; a limb bone } \text { (Eu 1.5.142) }^{1}\end{array}$ & $4780 \pm 90$ & $\begin{array}{c}-19.0 \\
\text { (assumed) }\end{array}$ & & & & 3710-3360 cal. вс & $\begin{array}{l}3655-3615 \text { cal. } \text { вс }(79 \%) \text { or } \\
3570-3530 \text { cal. } \text { BC }(16 \%)\end{array}$ \\
\hline OxA-449 & $\begin{array}{l}\text { Eu 1.5.143, parietal bone, from } \\
\text { disarticulated skull II of older male, } \\
\text { NW chamber primary deposit }\end{array}$ & $4825 \pm 90$ & $\begin{array}{c}-19.0 \\
\text { (assumed) }\end{array}$ & & & & 3790-3370 cal. вс & $\begin{array}{l}3660-3615 \mathrm{cal} . \text { Bс }(79 \%) \text { or } \\
3565-3530 \mathrm{cal} . \text { ВС }(16 \%)\end{array}$ \\
\hline OxA-12283 & $\begin{array}{l}\text { WK 21, disarticulated adult right } \\
\text { humerus from NW chamber primary } \\
\text { deposit }\end{array}$ & $4835 \pm 33$ & -19.9 & 11.0 & 3.1 & & 3670-3530 cal. вс & $\begin{array}{l}3655-3625 \mathrm{cal} . \text { BC }(79 \%) \text { or } \\
3560-3535 \mathrm{cal} . \text { BC }(16 \%)\end{array}$ \\
\hline OxA-13188* & $\begin{array}{l}\text { WK 22, disarticulated adult right } \\
\text { humerus from NW chamber primary } \\
\text { deposit }\end{array}$ & $4767 \pm 38$ & -20.4 & 9.4 & 3.3 & & 3650-3370 cal. вс & $\begin{array}{l}3650-3615 \mathrm{cal} . \text { вс }(79 \%) \text { or } \\
3570-3530 \mathrm{cal} . \text { ВС }(16 \%)\end{array}$ \\
\hline OxA-451 & $\begin{array}{l}38, \text { left femur from partially } \\
\text { articulated skeleton IV, sub-adult } \\
\text { c. } 16-20 \text { years, SW chamber primary } \\
\text { deposit }\end{array}$ & $4780 \pm 90$ & $\begin{array}{c}-19.0 \\
\text { (assumed) }\end{array}$ & & & & 3710-3360 cal. вс & $\begin{array}{l}3655-3615 \mathrm{cal} . \text { вс }(79 \%) \text { or } \\
3570-3530 \mathrm{cal} . \text { ВС }(16 \%)\end{array}$ \\
\hline OxA-13201* & $\begin{array}{l}\text { WK 15, foot phalange, skeleton } \\
\text { SW I, sub-adult, c. 10-14 years, SW } \\
\text { chamber primary deposit. Recovered } \\
\text { disarticulated, although sufficient } \\
\text { elements survive to suggest that } \\
\text { the individual was at least partially } \\
\text { articulated on deposition. }\end{array}$ & $4827 \pm 38$ & -20.6 & 9.5 & 3.1 & & $3670-3520$ cal. вс & $\begin{array}{l}3655-3620 \mathrm{cal} . \text { BC }(80 \%) \text { or } \\
3560-3535 \mathrm{cal} . \text { BC }(15 \%)\end{array}$ \\
\hline
\end{tabular}


Table 1. (cont.)

\begin{tabular}{|c|c|c|c|c|c|c|c|c|}
\hline $\begin{array}{l}\text { Laboratory } \\
\text { no. }\end{array}$ & Sample no. and material & $\begin{array}{l}\text { Radiocarbon } \\
\text { age (BP) }\end{array}$ & $\delta^{13} \mathrm{C}(\%)$ & $\begin{array}{c}\delta^{15} N \\
(\%)\end{array}$ & $\begin{array}{l}\mathrm{C}: \mathrm{N} \\
\text { ratio }\end{array}$ & $\begin{array}{l}\text { Weighted } \\
\text { mean (вP) }\end{array}$ & $\begin{array}{l}\text { Calibrated date } \\
\text { range }(95 \% \\
\text { confidence) }\end{array}$ & $\begin{array}{l}\text { Posterior density } \\
\text { estimate (probability) }\end{array}$ \\
\hline OxA-12284 & $\begin{array}{l}\text { WK 24, disarticulated adult right } \\
\text { humerus from SW chamber primary } \\
\text { deposit }\end{array}$ & $4797 \pm 31$ & -20.5 & 9.4 & 3.1 & & $3650-3520$ cal. вс & $\begin{array}{l}3650-3620 \text { cal. } B C(79 \%) \text { or } \\
3565-3530 \text { cal. } B C(16 \%)\end{array}$ \\
\hline OxA-13332* & $\begin{array}{l}\text { WK 25, disarticulated adult right } \\
\text { humerus from SW chamber primary } \\
\text { deposit }\end{array}$ & $4791 \pm 37$ & -21.1 & 9.8 & 3.1 & & 3650-3380 cal. вс & $\begin{array}{l}3655-3615 \text { cal. } B C(79 \%) \text { or } \\
3565-3530 \mathrm{cal} . \text { BC }(16 \%)\end{array}$ \\
\hline OxA-13190* & $\begin{array}{l}\text { WK 26, disarticulated adult right } \\
\text { humerus from SW chamber primary } \\
\text { deposit }\end{array}$ & $4680 \pm 39$ & -21.0 & 11.8 & 3.3 & & 3630-3360 cal. вс & $\begin{array}{l}3645-3605 \text { cal. } \text { вС }(80 \%) \text { or } \\
3570-3525 \text { cal. } \text { вС }(15 \%)\end{array}$ \\
\hline GrA-23180 & \multirow{2}{*}{$\begin{array}{l}\text { WK } 12 \text {, right femur from partially } \\
\text { articulated contracted skeleton NE III, } \\
\text { NE chamber primary deposit }\end{array}$} & $4790 \pm 50$ & -22.3 & 10.5 & & \multirow{2}{*}{$\begin{array}{l}4838 \pm 26 \\
T^{\prime}=1.3 \\
T^{\prime}(5 \%)=3.8\end{array}$} & \multirow{2}{*}{ 3660-3530 cal. вс } & \multirow{2}{*}{$\begin{array}{l}3655-3630 \mathrm{cal} . \text { вс }(81 \%) \text { or } \\
3560-3540 \mathrm{cal} . \text { ВС }(14 \%)\end{array}$} \\
\hline OxA-12652 & & $4856 \pm 31$ & -20.5 & 10.4 & 3.3 & & & \\
\hline OxA-450 & $\begin{array}{l}\text { Eu 1.5.140, left femur from articulated } \\
\text { skeleton II, adult male, NE chamber } \\
\text { primary deposit }\end{array}$ & $4700 \pm 80$ & $\begin{array}{c}-19.0 \\
\text { (assumed) }\end{array}$ & & & \multirow{2}{*}{$\begin{array}{l}4805 \pm 28 \\
T^{\prime}=1.9 \\
T^{\prime}(5 \%)= \\
3.8 ; v=1\end{array}$} & \multirow[t]{2}{*}{ 3650-3520 cal. вс } & \multirow[t]{2}{*}{$\begin{array}{l}3650-3620 \mathrm{cal} . \text { BC }(79 \%) \text { or } \\
3560-3530 \mathrm{cal} . \text { BC }(16 \%)\end{array}$} \\
\hline OxA-12282 & $\begin{array}{l}\text { WK } 20 \text {, right femur, replicate of } \\
\text { OxA- } 450\end{array}$ & $4819 \pm 30$ & -20.2 & 10.6 & 3.1 & & & \\
\hline OxA-13198* & $\begin{array}{l}\text { WK 11, right femur from partially } \\
\text { articulated skeleton NE I, adult } \\
\text { female, NE chamber primary deposit }\end{array}$ & $4838 \pm 37$ & -20.5 & 9.6 & 3.1 & & 3700-3530 cal. вс & $\begin{array}{l}3655-3625 \mathrm{cal} . \text { BC }(80 \%) \text { or } \\
3560-3535 \mathrm{cal} . \text { BC }(15 \%)\end{array}$ \\
\hline OxA-13182* & $\begin{array}{l}\text { WK 6, tibia from infant, birth-six } \\
\text { months, SE chamber secondary } \\
\text { deposits; from a largely complete set } \\
\text { of post-cranial remains, suggesting } \\
\text { articulation or partial articulation at } \\
\text { the time of deposition }\end{array}$ & $4454 \pm 34$ & -19.3 & 11.0 & 3.4 & & 3340-2920 cal. вС & $\begin{array}{l}3345-3205 \mathrm{cal} . \text { BC }(88 \%) \text { or } \\
3195-3150 \mathrm{cal} . \text { BC }(7 \%)\end{array}$ \\
\hline OxA-13242* & $\begin{array}{l}\text { WK } 7 \text {, rib from foetus, } 5-7 \text { months in } \\
\text { utero, SE chamber secondary deposits; } \\
\text { from a partial skeleton, suggesting } \\
\text { that it was articulated on deposition }\end{array}$ & $4506 \pm 37$ & -20.1 & 11.5 & 3.1 & & 3360-3030 cal. вс & $\begin{array}{l}3300-3085 \text { cal. } B C(91 \%) \text { or } \\
3060-3030 \mathrm{cal} . \text { BC }(4 \%)\end{array}$ \\
\hline OxA-13184* & $\begin{array}{l}\text { WK 9, tibia from a child, c. 2-3 years, } \\
\text { from SE chamber secondary deposits; } \\
\text { from a largely complete skeleton, } \\
\text { suggesting articulation on deposition }\end{array}$ & $4478 \pm 37$ & -21.2 & 10.8 & 3.3 & & 3360-3020 cal. вС & 3305-3320 cal. BC (95\%) \\
\hline OxA-13183* & $\begin{array}{l}\text { WK } 8 \text {, humerus from an infant, } \\
12-18 \text { months, SE chamber secondary } \\
\text { deposits; from a partial skeleton, } \\
\text { suggesting that it was articulated on } \\
\text { deposition }\end{array}$ & $4103 \pm 38$ & -20.6 & 11.4 & 3.4 & & 2870-2490 cal. вС & $\begin{array}{l}2875-2800 \mathrm{cal} . \mathrm{BC}(41 \%) \text { or } \\
2780-2595 \mathrm{cal} . \mathrm{BC}(54 \%)\end{array}$ \\
\hline OxA-13181* & $\begin{array}{l}\text { WK 5, rib fragment of infant, } \\
\text { c. 12-18 months, SE chamber } \\
\text { secondary deposits. From largely } \\
\text { complete skeleton recovered on the } \\
\text { surface of the undisturbed chalk of the } \\
\text { secondary filling } \\
\text { The completeness of the skeleton } \\
\text { suggests articulation on deposition. }\end{array}$ & $4105 \pm 35$ & -20.5 & 14.0 & 3.3 & & 2870-2490 cal. вС & $\begin{array}{l}2840-2805 \mathrm{cal} . \text { BC }(4 \%) \text { or } \\
2760-2565 \mathrm{cal} . \text { BC }(87 \%) \text { or } \\
2525-2495 \mathrm{cal} . \text { BC }(4 \%)\end{array}$ \\
\hline OxA-13243* & $\begin{array}{l}\text { WK 10, humerus fragment from } \\
\text { infant, c. 6-10 months, NE chamber } \\
\text { secondary deposits } \\
\text { Most of the skeleton is present, } \\
\text { indicating articulation at the time of } \\
\text { deposition. }\end{array}$ & $4583 \pm 45$ & -20.9 & 13.7 & 3.1 & & 3500-3100 cal. вс & $\begin{array}{l}3495-3455 \mathrm{cal} . \text { BC }(4 \%) \text { or } \\
3380-3260 \mathrm{cal} . \text { BC }(34 \%) \text { or } \\
3250-3095 \mathrm{cal} . \text { BC }(57 \%)\end{array}$ \\
\hline OxA-13202* & $\begin{array}{l}\text { WK 17, tibia (Capra sp., male) from } \\
\text { a partially articulated skeleton, NW } \\
\text { chamber secondary deposits }\end{array}$ & $3934 \pm 36$ & -23.3 & 5.4 & 3.1 & & 2560-2300 cal. вС & $\begin{array}{l}2570-2515 \mathrm{cal} . \text { BC }(20 \%) \text { or } \\
2500-2335 \mathrm{cal} . \text { BC }(75 \%)\end{array}$ \\
\hline
\end{tabular}

methods outlined in Gillespie et al. (1984) and Wand et al. (1984). The series of samples dated at the Oxford Radiocarbon Accelerator Unit in 2001 and 2002 were processed using the gelatinization protocol described by Bronk Ramsey et al. (2000). Following the discovery in the laboratory of a contamination problem associated with this method, in eighteen cases the contaminated material was re-processed, graphitized and dated, as described by Bronk Ramsey et al. (2004a). These results are denoted by an asterisk in Table 1. All the other samples dated at Oxford were processed using collagen extraction (Law \& Hedges 1989; Hedges 


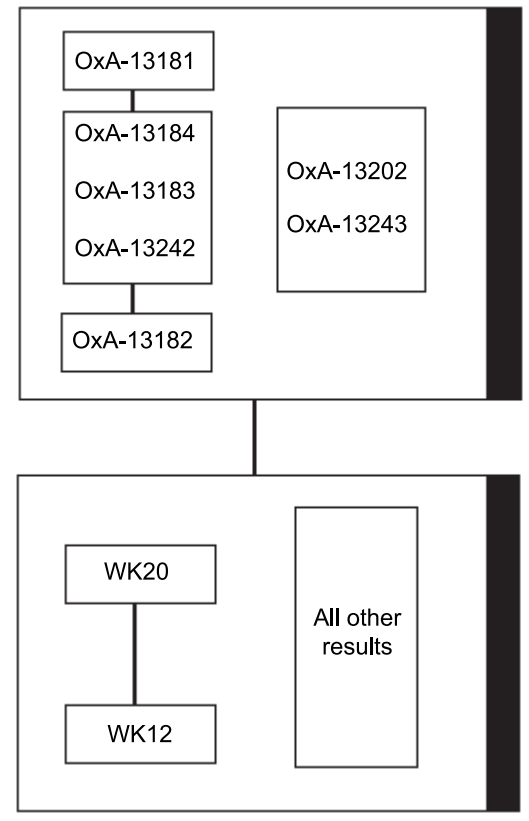

Figure 5. Summary of prior information incorporated in the chronological model shown in Figures 6 and 7. The stratigraphic relationships between samples are shown with the earliest at the bottom, and the solid bars down the right-hand side represent uniformly distributed phases of activity.

et al. 1989), followed by the revised gelatinization and filtration protocol described by Bronk Ramsey et al. (2004a), and dated by AMS as outlined in Bronk Ramsey et al. (2004b). In addition, four samples of human bone were dated by the Centre of Isotope Research at the Rijksuniversiteit Groningen in 2003. They were processed and measured as described by Aerts-Bijma et al. $(1997 ; 2001)$ and van der Plicht et al. (2000).

\section{Interpretations}

Two alternative chronological models for the West Kennet long barrow are shown in Figures 5-7, and Figures 8-10.

\section{Primary deposits}

A schematic representation of the composition of the primary deposits of human bone in each chamber is given in Figure 2, the result of recent re-analysis.

Six individuals were dated from the primary deposits in the southeast chamber. Two were dated in replicate by different AMS laboratories, in each case producing statistically consistent radiocarbon measurements (Table 1, WK 2 \& WK 4). All this material was disarticulated but there were sufficiently abundant ribs, vertebrae, hand and foot bones assign- able to five of these individuals to strongly suggest that they were probably in an articulated or partially articulated state when deposited. The sixth individual is represented by a disarticulated adult femur, and so we have no evidence whether this body was originally deposited articulated. From the published site photographs, it seems that this material, much of which appears to have been originally deposited in at least partial articulation, was subsequently disturbed and scattered about the chamber. Consequently, there are no certain stratigraphic relationships between the dated individuals in this chamber although some of the adult bones were recorded overlying some of the immature material (see Piggott 1962, pl. XVI).

Five individuals have been dated from the primary deposits in the northwest chamber. Two statistically inconsistent radiocarbon results (Table 1) have been obtained on the scapula from the partially articulated individual NW I (Table 1, WK16), although the difference between the two measurements is sufficiently small to suggest that one may simply be a statistical outlier. Therefore the measurements have been combined before calibration. Four other disarticulated bones were dated. Two right humeri, which on morphometric grounds do not belong to NW I, certainly represent two more individuals. It is less clear whether the two samples dated in 1984 represent another two people. The skull (NW II; OxA-449) is certainly not part of NW I (which has a perfectly good skull of its own!). The limb bone (OxA-563), originally tentatively attributed to NW I on spatial grounds (Wells 1962, 79), was probably not from that individual, on morphometric grounds. Either of these two samples, however, might be from one of the individuals whose right humerus has been dated.

Five individuals have also been dated from the primary deposits in the southwest chamber. A left femur was dated from a partially articulated sub-adult (SW IV; OxA-451), and a foot phalange from a second sub-adult was also dated (SW I; OxA-13201). Sufficient elements from this skeleton were recovered to suggest that it was at least partially articulated on deposition, although the remains were recovered disarticulated. Samples from three right humeri represent three adults. These bones were recovered disarticulated, and it is difficult reliably to assign further bones to these individuals. Consequently, we have no evidence on whether these skeletons were originally deposited in articulation.

Five radiocarbon results are available from three at least partially articulated skeletons in the northeast chamber. Two were dated in replicate, in each case producing statistically consistent radiocarbon meas- 
urements (Table 1, WK12 \& WK 20). Piggott $(1962,25)$ states clearly that burial NE II partly overlay burial NE III. However, the published photographic evidence (Piggott 1962, pls. XVa, XVb) suggests the opposite relationship. It is this latter sequence that has been incorporated in the chronological model, because there is little evidence, as far as one can tell from the photographs, of any human remains being stratified beneath NE II. The elements to the southwest of NE II's skull are too small to be the bones that Wells reports and that we have identified as belonging to NE III.

No samples were dated from the west chamber. Material almost certainly from the Thurnam excavations of this chamber does survive, having been recovered from Thurnam's spoil heap in the excavation of the 1950s (Wysocki \& Whittle in prep.), but given this history and its scarcity, it was decided not to use it in this programme. Four male crania (recently reexamined and confirmed as such by Wysocki) from this chamber are also extant as part of the Thurnam collection. These have all had extensive varnishing and reconstructive work and are quite unsuitable for radiocarbon dating.

\section{Secondary deposits}

Numerous sub-assemblages containing immature human bone, animal bone fragments, pieces and flecks of charcoal, rough flint flakes or small potsherds and white powdered chalk or larger chalk clasts and accretions were found in the archive labelled as coming from the secondary fill deposits. In other cases, where labelling was absent or uninformative, such sub-assemblages still contained fragmentary animal, flint, chalk, ceramic and carbonized material, again indicating a secondary fill deposit. Furthermore, bones in these assemblages were stained with a fine white chalky soil, whereas bone from the primary deposits was consistently stained with a dark brown soil. Consequently it was possible to allocate unlabelled or poorly labelled sub-assemblages to either primary or secondary contexts. It is clear that a considerable amount of human material from the secondary fill was never reported, or only partially reported by Piggott and colleagues. In the case of the southeast chamber, material from both primary and secondary deposits was reported by Wells (1962) without making any distinction between the two contexts (i.e. as if all remains came from the primary deposits).

The remains of five infants from the secondary filling of the southeast chamber have been dated. Only limited contextual information accompanied the skeletal archive, but most probably the earliest of these burials is WK 6 (OxA-13182). This was labelled as coming 'from dark rubble 4 ' below datum', apparently equivalent to layer 10 (Piggott 1962, 26-7, fig. 9). WK 7, WK 8 and WK 9 (OxA-13242, OxA-13183, and OxA-13184) are likely to be stratigraphically later than this, from within layer 2. Of these, WK 8 (OxA13183) may be the latest as it is likely to be from the upper part of layer 2, because the labelling says that it is 'from above the dry stone walling of the northwest corner of the southeast chamber'. The precise locations of WK 7 and WK 9, however, are not known and so this relationship is not included in the chronological model. WK 5 (OxA-13181) is the latest of the dated samples in this chamber, coming 'from the surface of the undisturbed chalk', equivalent to the surface of layer 2.

A substantially complete skeleton of an infant provided the single sample from the secondary fill of the northeast chamber (WK 10, OxA-13243). This came from a context labelled simply as 'upper layer'.

A largely complete, and partially articulated, goat skeleton from the middle or upper part of layer 3 in the northwest chamber (Piggott 1962, fig. 9; WK 17 , OxA-13202) provides the final sample from the secondary fills.

\section{Results from chronological modelling}

The model shown in Figures 6 and 7 suggests that the construction of the transepted monument at West Kennet, as dated from the primary mortuary deposits, occurred in 3670-3635 cal. BC (81\% probability) or 3575-3545 cal. вС (14\% probability: start primary). The last interments of this initial use of the burial chambers occurred in 3640-3610 cal. BC (77\% probability) or 3550-3520 cal. вс (18\% probability: end primary). The difference between these two distributions suggests that this primary mortuary activity continued for

Table 2. Posterior density estimates for the dates of archaeological events and the duration of activities at West Kennet, derived from the model described in Figures 5-7.

\begin{tabular}{|l|l|l|}
\hline & \multicolumn{2}{|c|}{ Model 1 (Figs. 5-7) } \\
\hline Distribution & $\begin{array}{l}\text { Posterior density } \\
\text { estimate } \mathbf{6 8 \%} \\
\text { probability) }\end{array}$ & $\begin{array}{l}\text { Posterior density estimate } \\
\text { (95\% probability) }\end{array}$ \\
\hline start primary & $3655-3635 \mathrm{cal}$. BC & $\begin{array}{l}3670-3635 \text { cal. BC }(81 \%) \text { or } \\
3575-3545 \text { cal. BC }(14 \%)\end{array}$ \\
\hline end primary & $3635-3615 \mathrm{cal}$. BC & $\begin{array}{l}3640-3610 \text { cal. BC }(77 \%) \text { or } \\
3550-3520 \text { cal. BC }(18 \%)\end{array}$ \\
\hline start infill & $3510-3295 \mathrm{cal}$. BC & $3620-3240$ cal. BC \\
\hline end infill & $2475-2225$ cal. BC & $2545-2065$ cal. BC \\
\hline primary_use & $10-30$ years & $\begin{array}{l}1-55 \text { years }(94 \%) \text { or } \\
115-140 \text { years }(1 \%)\end{array}$ \\
\hline abandonment & $95-320$ years & $1-375$ years \\
\hline infill & $895-1235$ years & $775-1420$ years \\
\hline
\end{tabular}




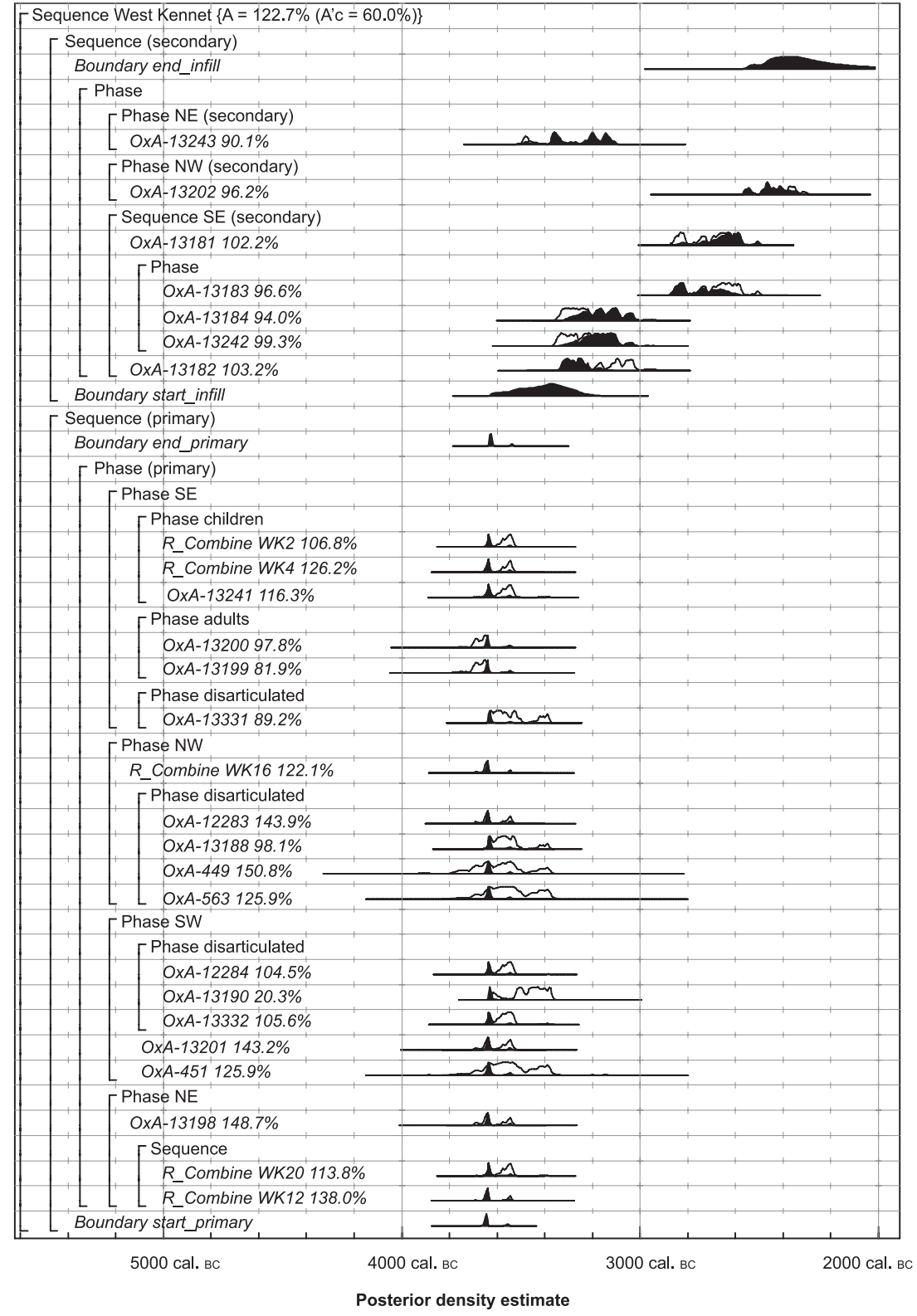

Figure 6. Probability distributions of dates from West Kennet, with all burials in the primary mortuary deposits interpreted as freshly dead individuals. The format is identical to that in Figure 4. The large square brackets down the left-hand side and the OxCal keywords define the overall model exactly.

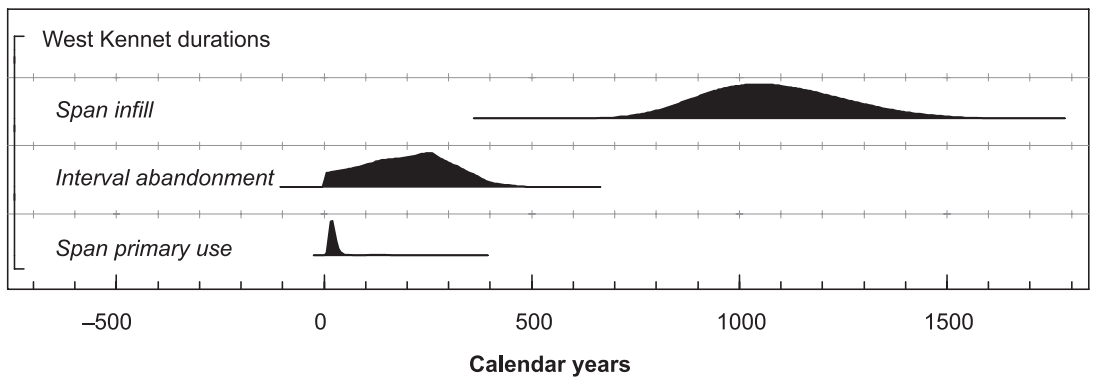

only $10-30$ years (68\% probability), 1-55 years (94\% probability) or 115-140 years ( $1 \%$ probability: primary use) (see also Table 2).

The short duration of the primary mortuary activity at West Kennet was not anticipated. Using radiocarbon dating, it is not possible to distinguish any chronological variation in the spatial distribution of corpses within chambers, or between chambers, when the actual duration of the activity was so short.

The radiocarbon determinations on all 19 dated individuals from the primary mortuary deposit are statistically consistent $\left(T^{\prime}=26.4 ; T^{\prime}(5 \%)=\right.$ $28.9 ; v=18$ ) (Ward \& Wilson 1978). This means that all these individuals could have died at the same time, although they do not have to be precisely contemporary. It is likely, however, that they are all close in date.

The chronological model shown in Figures 6 and 7 shows good overall agreement $\left(\mathrm{A}_{\text {overall }}=122.7 \%\right)$, as the radiocarbon dates are consistent with the interpretation, included in the model, that none of the disarticulated material was residual or ancestral. Given that the radiocarbon measurements from these deposits form such a coherent group, if the disarticulated samples were ancestral by even one or two generations, the model would probably show poor agreement (see below for further discussion of this point). The model shown also has good convergence (Bronk Ramsey 1995; and see Bayliss et al. this issue). ${ }^{2}$

Once the primary mortuary deposition had finished, there seems to have been a hiatus before the secondary accumulation of deposits within the chambers began. This hiatus probably lasted for rather more

Figure 7. Probability distributions of the number of years during which various activities occurred in the West Kennet long barrow, derived from the model shown in Figure 5. 
than a century (Figs. 6 \& 9: abandonment). According to the model shown in Figures 5 and 6, the infilling of the chambers began in $3620-3240$ cal. BC $(95 \%$ probability: start infill), and continued into the second half of the third millennium cal. вс (Fig. 6: end infill). Overall the infilling of the chambers took around a thousand years (Fig. 6: infill). We discuss the archaeological significance of these issues further below.

An alternative model for the chronology of the monument is shown in Figures 8-10. In this case, we have not assumed that all the people dated from the primary mortuary deposits were placed in the monument in a fleshed or partially articulated condition. They could therefore have died before the monument was built, although they must have died before the end of the formation of the mortuary deposit (Fig. 8). This interpretation is included in the second model (Figs. 9 \& 10), which also shows good overall agreement $\left(\mathrm{A}_{\text {overall }}=83.1 \%\right)$. This model has poor convergence, however (Bronk Ramsey 1995, 429). This means that it is unstable and does not produce consistent results.

This can be illustrated by more detailed consideration of the posterior density estimate for the start of deposition of the primary burials in the chambers (start primary: Fig. 9). This distribution is bi-modal because of the strong 'wiggle' in the calibration curve between 3620 and 3540 cal. вс (Reimer et al. 2004; Bayliss et al. this issue, p. 18, fig. 18). The problem is that the MCMC sampler tends to get trapped on one of the peaks, and so is not able to consider adequately the possibility that the actual date of the distribution lies on the other peak. This is illustrated in Figure 11, where the sampler has become trapped in the earlier part of the thirty-seventh century cal. BC, even though it is more likely that start primary really dates to the later peak centring on the 3550s.

For this reason, it is not valid to quote the posterior density estimates derived from this alternative model. For example, the relative probabilities that start primary falls on the earlier peak in the mid-thirtyseventh century or on the later peak in the mid-thirtysixth century vary significantly between runs of the model. Consistently, however, this interpretation of the primary deposits at West Kennet favours the later peak, and again a very short span of use is always preferred. ${ }^{3}$

We believe on archaeological and osteological grounds (discussed above with reference to the probable absence of secondary rites) that, of these two models, the first is the more plausible. Missing bones and disarticulation are the only reasons for supposing that there could have been secondary burial at West Kennet, but it is clear that disarticulation is the result

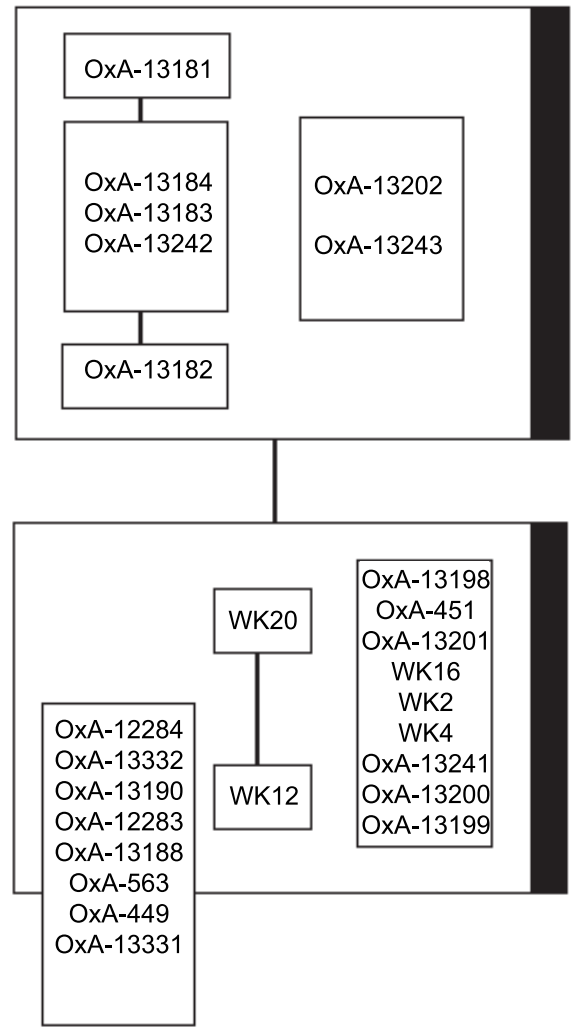

Figure 8. Summary of prior information incorporated in the chronological model shown in Figures 9 and 10. The stratigraphic relationships between samples are shown with the earliest at the bottom, and the solid bars down the right-hand side represent uniformly distributed phases of activity.

at least in part of movement of material around the chambers, as seen in the rearrangement of material in the northwest chamber (e.g. caches of vertebrae: Piggott 1962, fig. 8). It is also clear that there are a number of possible post-depositional, excavational and post-excavational factors that could also account for missing bones (Wysocki \& Whittle in prep.). We can note that Piggott $(1962,67)$ also thought secondary burial unlikely here.

\section{Discussion}

The dating programme at West Kennet was slightly more limited than those for Hazleton long cairn and the Ascott-under-Wychwood, Fussell's Lodge and Wayland's Smithy long barrows, reported in this series, for which samples constraining the constructions were available. An archaeologically more reliable model for West Kennet would require samples from a wider range of contexts, including the buried soil underlying the barrow, the primary sarsen core and 


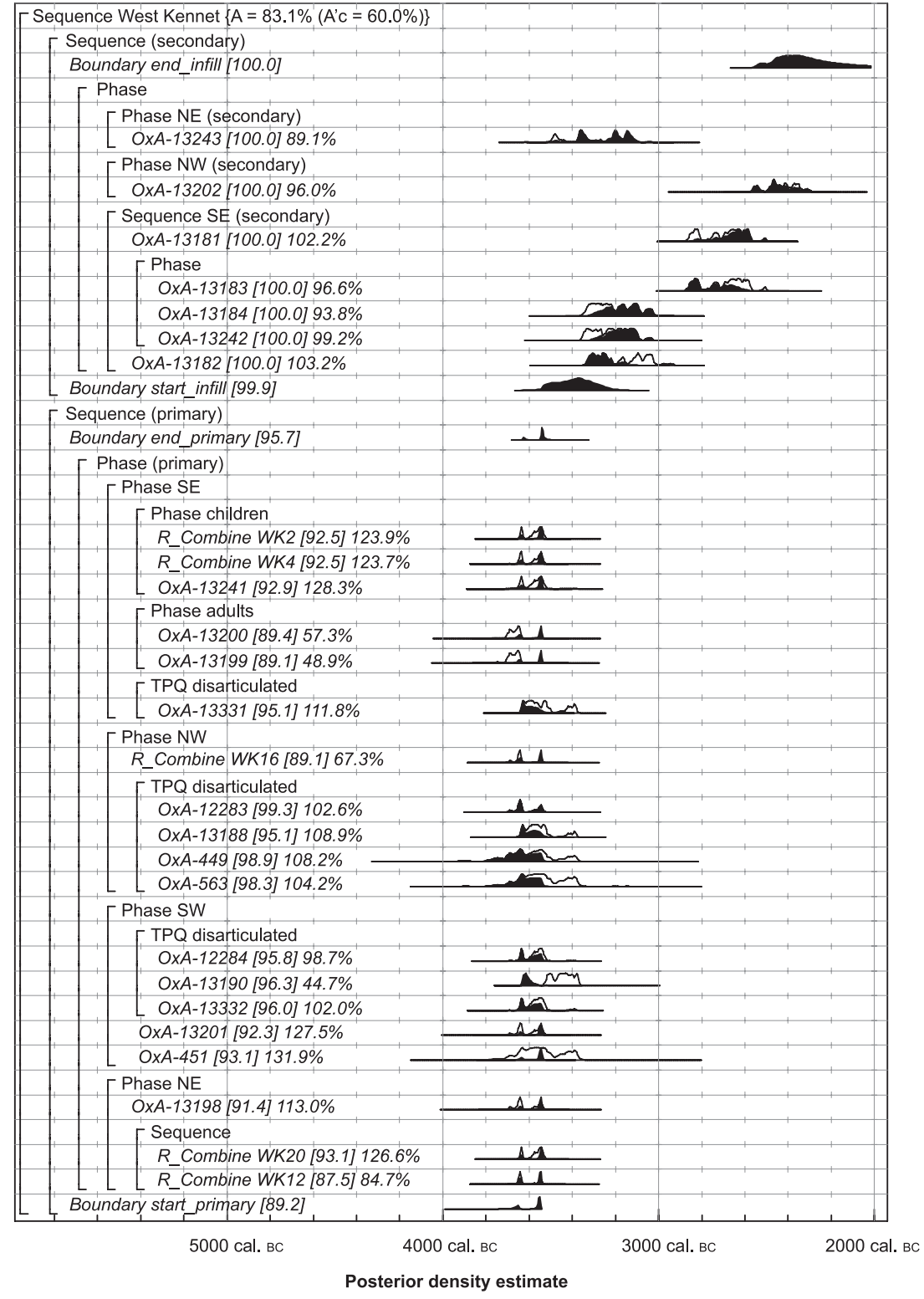

Figure 9. Probability distributions of dates from West Kennet, following an alternative model, with disarticulated single bones from the primary mortuary deposits interpreted as potential older, secondary depositions. The format is identical to that in Figure 4, with the convergence for each posterior density estimate given in square brackets. The large square brackets down the left-hand side and the OxCal keywords define the overall model exactly.



the fills of the flanking ditches. Those contexts have already been excavated on a small scale (Piggott 1962) and it would not require great effort for such trenches to be re-opened or extended at some point in the future.

The qualifications must therefore be stressed that the dates obtained here are termini ante quos for the construction of the monument, in the form that encompassed the chambers, and that we are assuming that bodies were deposited in the chambers as soon as or very soon after those had been constructed. We cannot discount the possibility that the monument began with a smaller construction (as at Wayland's Smithy), as already noted above, nor entirely disprove the possibility that other bodies or human remains had earlier been deposited in the chambers but had been subsequently cleared out. However, it is demonstrated above that there is no support for the disarticulated remains here being any older than the articulated, or partially articulated, remains.

On the basis of the main model presented above, we can now date the construction of the monument to the middle decades of the thirtyseventh century cal. вс (3670-3635 cal. $B C$ at $81 \%$ probability). There is, however, some possibility that the monument was actually built in the second quarter of the thirty-sixth century cal. вс (3575-3745 cal. вс at $14 \%$ probability). We believe that the alternative model, which perhaps favours this later construction date, is less plausible on archaeological and osteological grounds. Compared with what has been so often suggested in the literature, the results suggest a surprisingly short span of primary use (10-30 years at $68 \%$ probability or

Figure 10. Probability distributions of the number of years during which various activities occurred in the West Kennet long barrow, derived from the model shown in Figure 8. 
1-55 years at 94\% probability: primary use). That our attention now needs to be given to the changing mindsets of particular people in particular places and times is reinforced by the succeeding gap of rather more than a century (abandonment); the intensive activity of people in perhaps the 3640s or 3630s is highlighted by the inactivity - at least in this domain - of their successors. This model contrasts now rather strongly with the view of Piggott (1962) that the primary phase of the monument lasted some centuries, and was followed by a long hiatus before the eventual supposed quick secondary deposition. One further clue to the reality of an abandonment may be found in the signs of decay, represented by the partial collapse of portions of the drystone walling and blocking (Piggott 1962, 26-9), which took place before the introduction of the secondary deposits. By this stage, this was a monument whose fabric and contents were no longer at the forefront of people's minds, or no longer accessible.

Though this account has differed in many important details from that of Stuart Piggott, it is worth stressing that the recent work was only possible thanks to the quality of the excavations at West Kennet. We can note also that, in terms of the total duration of the monument, Piggott's final verdict on timespan was surprisingly good in an era when radiocarbon dating had scarcely been established, since he ended his report by stating that 'it seems unavoidable that the use of the West Kennet tomb, from building to final blocking, can hardly have spanned less than a millennium' (1962, 78).

We will take the later history of the monument first. The results presented here strongly suggest that Piggott's interpretation of the secondary deposits as the result of a single, final act as the culmination of a long history is incorrect. The dates now available indicate gradual deposition, over a period of centuries (infill). If all the material had been accumulated elsewhere (even if only in the forecourt) over a long period of time and then had been deposited quickly in the chambers and passage, we would not expect the chronologically coherent stratigraphic sequence demonstrated in Figure 6. One could expect, on Piggott's model, to find latest material at the base of the infill. The results support the alternative model suggested by Thomas \& Whittle (1986), though it is worth pointing out again that an archaeologically more reliable model in this instance would have required more samples from more contexts in the chambers. The deposits in question are not now available for further investigation. Future research here, however, could exploit the dating possibilities of sherds with organic residues and of calcined bone. The claim for a chronologically coherent stratigraphic sequence in the secondary deposits recalls the suggestion by Humphrey Case $(1995,11)$ that these had been inserted from above, after the partial removal of the capstones. This is further supported by the observation that chamber entrance blocking stones in the southwest chamber had collapsed on to the primary deposits and were overlain by the earliest layers of secondary infill (Piggott 1962, 26; Fig. 9). This small detail may also give us cause to consider the nature of the hiatus or abandonment. It is clear that the blocking of the entrances to the individual chambers took place before infilling. The collapse of a portion of this blocking in the southwest chamber suggests the passage of some time. It is possible that the blocking of the entrances to the chambers took place at the beginning of the period of abandonment, shortly after the last primary interments had been deposited. Rather than abandonment in the sense of an unplanned retreat in the face of contrary circumstances, there may have been a more formal or deliberate closure, perhaps also involving the erection of the blocking sarsens in the forecourt.

Looking beyond the monument itself, the later parts of the local sequence were rather imprecisely defined after fieldwork undertaken between 1987 and 1993 (Whittle 1993; 1994; 1997), and little further precision has been gained since then (Pollard \& Reynolds 2002; Gillings \& Pollard 2004; Pitts 2001). We are still unsure of the dates of the major monuments of Avebury and Silbury Hill, though programmes are underway to rectify this; and the spread of dates from the West Kennet palisade enclosures (Whittle 1997) remains rather alarming. If the process of secondary infilling at the West Kennet long barrow lasted some centuries, it may have run from before the major earthwork enterprises of the Late Neolithic to some point during their development. The latest date achieved in the current programme at West Kennet long barrow is a little before 2400 cal. $\mathrm{BC}$, although this sample may well not represent the latest activity in the process of secondary filling. The posterior density estimate for the articulated goat skeleton in the middle or upper part of layer 3 in the NW chamber of the secondary infilling is $2570-2515$ cal. вС (20\% probability) or 2500 2335 cal. BC (75\% probability; OxA-13202); the southeast chamber (on the basis of OxA-13181, a largely complete infant from the surface of the secondary filling) may have been filled earlier in the third millennium cal. вс. The date from the goat is not inconsistent with the currency of Beakers nationally but, given the continuing uncertainties about Beaker sequences and chronology (e.g. Case 1993), we need to keep an open mind on details; and, as noted above, the date of the 


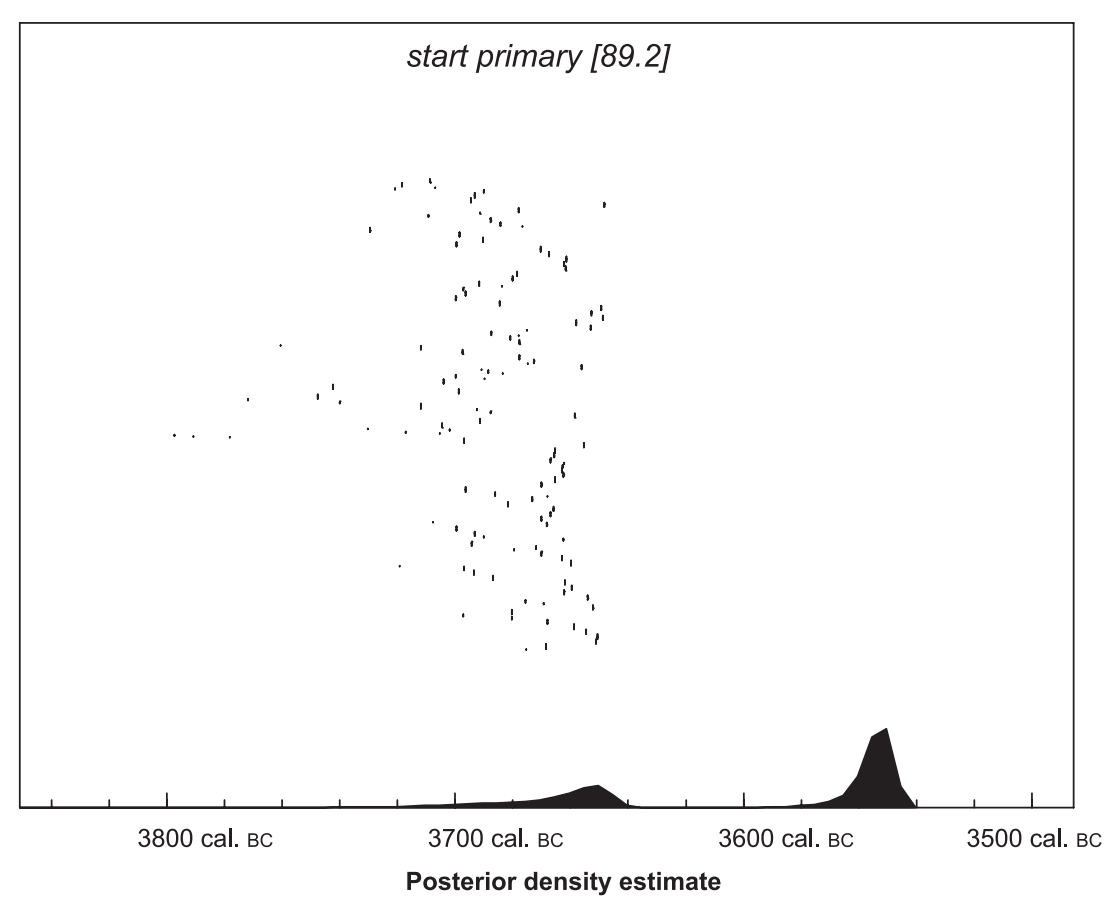

Figure 11. Convergence data for the posterior density estimate 'start primary' from the model shown in Figures 8 and 9. Each dot represents a single sample. This is only a small section of the total sampling run but allows one to see whether the model is concentrating on particular parts of the distribution.

final infilling here could in fact be a little later than the death of the goat. A more site-specific question remains the source of the main material constituting the secondary fill itself. Later material could be envisaged as coming from any number of constructions, but there are few if any signs of activity at monuments in the area when the process of secondary filling began. Rather than Piggott's suggested single source, we may have to think of a number of other places from which this material could have been drawn.

Turning back finally to the early history of the West Kennet long barrow in its regional context, the results presented here provide a first opportunity to reconsider the chronology of the early part of the Neolithic in the local region. A series of six rather broadly defined phases were proposed by Whittle after the fieldwork of 1987-93 (Whittle 1993; 1994, table 7). To Phase A (5450-5150 вр, с. 4300-3950 cal. вс) were tentatively assigned the first beginnings and clearances, and to Phase B (5150-4850 вр, c. 3950-3650 cal. $\mathrm{BC})$ some more signs of occupation and the first barrows. In Phase C (4850-4550 вр, с. 3650-3350 cal. вс) came more evidence for clearance and occupation in the form of lithic scatters and small pit groups, larger and more elaborate barrows, and the appearance of causewayed enclosures. This kind of scheme was later extended to other river valleys in central-southern England (Barclay 2000; 2006). The suggested phases for the Avebury area now seem too imprecisely defined, and they remain based on too few dates from too few sites. Other reviews of the evidence have also had to work within very broad and imprecise timescales (e.g. Pollard \& Reynolds 2002). While a trend may have been detected, it now seems more useful to set named phases aside and to concentrate on developments in terms of their absolute chronology. In this region as elsewhere in central-southern England, there is very little monumentality that can be placed before $c .3800 \mathrm{cal}$. BC. West Kennet long barrow can now strongly be suggested as belonging to the thirty-seventh century cal. вс. Other potentially earlier long barrows in the area (such as Horslip and South Street: Ashbee et al. 1979) are dated by far fewer determinations, and so far, their results have not been treated within a Bayesian statistical framework. Millbarrow, on the basis of its existing radiocarbon dates, could be seen as later than West Kennet, in the 'mid to later fourth millennium BC' (Whittle 1994, 46). On the basis of the results available after the 1988 excavations, the Windmill Hill causewayed enclosure was suggested as belonging to the 'middle of the fourth millennium BC' (Ambers \& Housley 1999, 119), and a visual (non-Bayesian) inspection of the results (Ambers \& Housley 1999, fig. 99) would be compatible with a period of use for the enclosure from before 3600 cal. BC to after 3400 cal. BC. Further results can be expected from a new programme of radiocarbon dating and Bayesian analysis covering causewayed enclosures in general, funded by English Heritage and the Arts and Humanities Research Council, and currently being conducted by Alex Bayliss, Frances Healy and Alasdair Whittle; these should allow us to offer far better precision for Windmill Hill, and to compare it in detail to West Kennet long barrow. The results from West Kennet in a real sense change everything for this region, and we will need to construct other detailed sequences for individual sites and monuments if we want to grasp more nuanced histories.

Some elements of such histories are already familiar, and much discussed, including an interest in the human dead and their arrangement, categorization 
and transformation; the monumentalization of the associated structure; and the emphasis given by this process to a specific place. What the results for the West Kennet long barrow presented here add, using our preferred first model, is a sense of the potential swiftness of change, the concentration of primary activity over a short period of time, and an interest not in timeless, generalized or anonymous forebears but in the dead of known, remembered and countable generations. This is a history which begins to speak for local agency and local identity, within the structure of wider changes elsewhere, a relationship which we consider in the final paper here (Whittle et al. this issue). Our sense of this narrative is becoming more specific, more contextualized and more personalized, and it is hard not to believe that it was similar for the Neolithic people involved.

\section{Acknowledgements}

The radiocarbon dating programme reported here was funded by English Heritage. We thank Amanda Chadburn for every assistance. We are grateful to the staff of the Oxford Radiocarbon Accelerator Unit and the Centre of Isotope Research, Rijksuniversiteit Groningen, for dating these samples. The re-analysis of the human bone assemblage was undertaken as part of a project funded by the Leverhulme Trust. We are grateful to Dr Robert Foley and Maggie Bellatti of the Duckworth Laboratory, Cambridge, for permission to examine and sample material from their collection. Finally, thanks to Andrew David, Jonathan Last, Peter Marshall, Mike Parker Pearson, Joshua Pollard and Julian Thomas for perceptive and constructive criticism of an earlier draft.

\section{Notes}

1. It seems that the left femur attributed to this skeleton by Wells $(1962,84)$ was dated, as this bone is now missing and there is no evidence of sampling on any of the other bones attributed to NW I. All the other bones sampled in 1984 are also missing.

2. It should be noted that some of the runs of this model have poor convergence, with some of the distributions from the primary mortuary deposits falling slightly below the $95 \%$ threshold. This is because the estimates for these dates at West Kennet are strongly bi-modally distributed, and on occasion the Metropolis-Hastings sampler is unable to determine whether the actual age of these samples falls in the mid-thirty-seventh or mid-thirty-sixth century cal. BC.

3. A later date for the construction of West Kennet, in the mid-thirty-sixth century cal. $\mathrm{BC}$, is also slightly more probable in a model which does not allow for the possibility of secondary deposition but in which the sequence of NE II and NE III is as described by Piggott (1962). This model also has consistently poor convergence and so is unstable, although an earlier date in the mid-thirty-seventh century cal. вс is always more probable than a later date in the mid-thirty-sixth century cal. вс.

\section{References}

AAHRG, 2001. Archaeological Research Agenda for the World Heritage Site. Salisbury: Avebury Archaeological and Historical Research Group.

Aerts-Bijma, A.T., H.A.J. Meijer \& J. van der Plicht, 1997. AMS sample handling in Groningen. Nuclear Instruments and Methods in Physics Research B 123, 221-5.

Aerts-Bijma, A.T., J. van der Plicht \& H.A.J. Meijer, 2001. Automatic AMS sample combustion and $\mathrm{CO}_{2}$ collection. Radiocarbon 43(2A), 293-8.

Ambers, J. \& R. Housley, 1999. Radiocarbon dating, in The Harmony of Symbols: the Windmill Hill Causewayed Enclosure, Wiltshire, eds. A. Whittle, J. Pollard \& C. Grigson. Oxford: Oxbow, 116-20.

Ashbee, P., I.F. Smith \& J.G. Evans, 1979. Excavation of three long barrows near Avebury, Wiltshire. Proceedings of the Prehistoric Society 45, 207-300.

Barclay, A., 2000. Spatial Histories of the Neolithic: a Study of the Monuments and Material Culture of Central Southern England. Unpublished PhD thesis, Department of Archaeology, University of Reading.

Barclay, A., 2006. Connections and networks: a wider world and other places, in Building Memories: the Neolithic Cotswold Long Barrow at Ascott-under-Wychwood, Oxfordshire, eds. D. Benson \& A. Whittle. Oxford: Oxbow, 331-44.

Bayliss, A. \& C. Bronk Ramsey, 2004. Pragmatic Bayesians: a decade integrating radiocarbon dates into chronological models, in Tools for Constructing Chronologies: Tools for Crossing Disciplinary Boundaries, eds. C.E. Buck \& A.R. Millard. London: Springer, 25-41.

Bayliss, A., C. Bronk Ramsey \& F.G. McCormac, 1997. Dating Stonehenge, in Science and Stonehenge, eds. B. Cunliffe \& C. Renfrew. Oxford: British Academy, 39-59.

Bayliss, A., C. Bronk Ramsey, J. van der Plicht \& A. Whittle, this issue. Bradshaw and Bayes: towards a timetable for the Neolithic.

Bronk Ramsey, C., 1995. Radiocarbon calibration and analysis of stratigraphy. Radiocarbon 36, 425-30.

Bronk Ramsey, C., 1998. Probability and dating. Radiocarbon 40, 461-74.

Bronk Ramsey, C., 2001. Development of the radiocarbon calibration program. Radiocarbon 43, 355-63.

Bronk Ramsey, C., P.B. Pettitt, R.E.M. Hedges, G.W.L. Hodgins \& D.C. Owen, 2000. Radiocarbon dates from the Oxford AMS system: Archaeometry datelist 30. Archaeometry 42, 459-79.

Bronk Ramsey, C., T.F.G. Higham, A. Bowles \& R.E.M. Hedges, 2004a. Improvements to the pre-treatment of bone at Oxford. Radiocarbon 46, 155-63.

Bronk Ramsey, C., T. Higham \& P. Leach, 2004b. Towards high precision AMS: progress and limitations. Radiocarbon 46, 17-24.

Case, H., 1993. Beakers: deconstruction and after. Proceedings 
of the Prehistoric Society 59, 241-68.

Case, H., 1995. Some Wiltshire Beakers and their contexts. Wiltshire Archaeological and Natural History Magazine $88,1-17$.

Corcoran, J.X.W.P., 1969. The Cotswold-Severn group. 1. Distribution, morphology and artefacts, in Megalithic Enquiries in the West of Britain, eds. T.G.E. Powell, J.X.W.P. Corcoran, F. Lynch \& J.G. Scott. Liverpool: Liverpool University Press, 13-72.

Darvill, T.C., 1982. The Megalithic Chambered Tombs of the Cotswold-Severn Region. Highworth: Vorda.

Evans, J.G., 1972. Land Snails in Archaeology. London: Seminar Press.

Gibson, A., 2000. Survey and Excavation at the West Kennet Long Barrow. (Centre for Archaeology Project Design 657 (unpublished).) Portsmouth: English Heritage.

Gillespie, R., R.E.M. Hedges \& J.O. Wand, 1984. Radiocarbon dating of bone by Accelerator Mass Spectrometry. Journal of Archaeological Science 11, 165-70.

Gillespie, R., J.A.J. Gowlett, E.T. Hall, R.E.M. Hedges \& C. Perry, 1985. Radiocarbon dates from the Oxford AMS system: Archaeometry datelist 2. Archaeometry 27, 237-46.

Gillings, M. \& J. Pollard, 2004. Avebury. London: Duckworth.

Gowlett, J.A.J., E.T. Hall \& R.E.M. Hedges, 1986a. The date of the West Kennet long barrow. Antiquity 60, 143-4.

Gowlett, J.A.J., R.E.M. Hedges, I.A. Law \& C. Perry, 1986 b. Radiocarbon dates from the Oxford AMS system: Archaeometry datelist 4. Archaeometry 28, 206-21.

Haglund, W.D., 1997a. Dogs and coyotes: postmortem involvement with human remains, in Forensic Taphonomy: the Postmortem Fate of Human Remains, eds. W.D. Haglund \& M.H. Sorg. Boca Raton (FL): CRC Press, 367-81.

Haglund, W.D., 1997b. Scattered skeletal human remains: search strategy considerations for locating missing teeth, in Forensic Taphonomy: the Postmortem Fate of Human Remains, eds. W.D. Haglund \& M.H. Sorg. Boca Raton (FL): CRC Press, 383-94.

Hedges, R.E.M., C.R. Bronk \& R.A. Housley, 1989. The Oxford accelerator mass spectrometry facility; technical developments in routine dating. Archaeometry 31, 99-113.

Law, I.A. \& R.E.M. Hedges, 1989. A semi-automated pretreatment system and the pretreatment of older and contaminated samples. Radiocarbon 31, 247-53.

Mant, A.K., 1987. Knowledge acquired from post-War exhumations, in Death, Decay and Reconstruction, eds. A. Boddington, A.N. Garland \& R.C. Janaway. Manchester: Manchester University Press, 65-80.

Martin, L., 2001. The West Kennet Long Barrow, Wiltshire: Report on Geophysical Survey, January 2001. (Centre for Archaeology Report 78/2001.) Portsmouth: English Heritage.

North, J., 1996. Stonehenge: Neolithic Man and the Cosmos. London: HarperCollins.

Pearson, G.W., J.R. Pilcher, M.G.L., Baillie, D.M. Corbett \& F. Qua, 1986. High-precision ${ }^{14} \mathrm{C}$ measurement of
Irish oaks to show the natural ${ }^{14} \mathrm{C}$ variations from $\mathrm{AD}$ 1840-5210 вс. Radiocarbon 28, 911-34.

Piggott, S., 1962. The West Kennet Long Barrow: Excavations 1955-56. London: Her Majesty's Stationery Office.

Pitts, M., 2001. Excavating the Sanctuary: new investigations on Overton Hill, Avebury. Wiltshire Archaeological and Natural History Magazine 94, 1-23.

Pollard, J. \& A. Reynolds, 2002. Avebury: the Biography of a Landscape. Stroud: Tempus.

Reimer, P.J., M.G.L. Baillie, E. Bard, A. Bayliss, J.W. Beck, C.J.H. Bertrand, P.G. Blackwell, C.E. Buck, G.S. Burr, K.B. Cutler, P.E. Damon, R.L. Edwards, R.G. Fairbanks, M. Friedrich, T.P. Guilderson, A.G. Hogg, K.A. Hughen, B. Kromer, F.G. McCormac, S. Manning, C. Bronk Ramsey, R.W. Reimer, S. Remmele, J.R. Southon, M. Stuiver, S. Talamo, F.W. Taylor, J. van der Plicht \& C.E. Weyhenmeyer, 2004. IntCal04 Terrestrial Radiocarbon Age Calibration, 0-26 cal kyr вр. Radiocarbon 46, 1029-58.

Roksandić, M., 2002. Position of skeletal remains as a key to understanding mortuary behaviour, in Advances in Forensic Taphonomy: Method, Theory and Archaeological Perspectives, eds. W.D. Haglund \& M.H. Sorg. Boca Raton (FL): CRC Press, 99-117.

Saville, A., 1990. Hazleton North: the Excavation of a Neolithic Long Cairn of the Cotswold-Severn Group. London: English Heritage.

Smith, I.F., 1965. Windmill Hill and Avebury. Oxford: Clarendon Press.

Stuiver, M. \& H.A. Polach, 1977. Reporting of ${ }^{14} \mathrm{C}$ data. Radiocarbon 19, 355-63.

Stuiver, M. \& P.J. Reimer, 1986. A computer program for radiocarbon age calculation. Radiocarbon 28, 1022-30.

Stuiver, M. \& P.J. Reimer, 1993. Extended ${ }^{14} \mathrm{C}$ data base and revised CALIB $3.0{ }^{14} \mathrm{C}$ age calibration program. Radiocarbon 35, 215-30.

Stuiver, M., P.J. Reimer, E. Bard, J.W. Beck, G.S. Burr, K.A. Hughen, B. Kromer, F.G. McCormack, J. van der Plicht \& M. Spur, 1998. INTCAL98 radiocarbon age calibration, 24,000-0 cal вр. Radiocarbon 40, 1041-84.

Thomas, J. \& A. Whittle, 1986. Anatomy of a tomb - West Kennet revisited. Oxford Journal of Archaeology 5, 129-56.

Thurnam, J., 1860. On the examination of a chambered long barrow at West Kennet, Wiltshire. Archaeologia $38,405-21$.

van der Plicht, J., S. Wijma, A.T. Aerts, M.H. Pertuisot \& H.A. Meijer, 2000. Status report: the Groningen AMS facility. Nuclear Instruments and Methods in Physics Research B 172, 58-65.

Wand, J.O., R. Gillespie \& R.E.M. Hedges, 1984. Sample preparation for Accelerator-based radiocarbon dating. Journal of Archaeological Science 11, 159-63.

Ward, G.K. \& S.R. Wilson, 1978. Procedures for comparing and combining radiocarbon age determinations: a critique. Archaeometry 20, 19-31.

Wells, L.H., 1962. The inhumation burials, in The West Kennet Long Barrow: Excavations 1955-56, ed. S. Piggott. London: Her Majesty's Stationery Office, 79-89. 
Whittle, A., 1993. The Neolithic of the Avebury area: sequence, environment, settlement and monuments. Oxford Journal of Archaeology 12, 29-53.

Whittle, A., 1994. Excavations at Millbarrow chambered tomb, Winterbourne Monkton, north Wiltshire. Wiltshire Archaeological and Natural History Magazine 87, 1-53.

Whittle, A., 1997. Sacred Mound, Holy Rings. Silbury Hill and the West Kennet Palisade Enclosures: a Later Neolithic
Complex in North Wiltshire. Oxford: Oxbow.

Whittle, A., A. Barclay, A. Bayliss, L. McFadyen, R. Schulting \& M. Wysocki, this issue. Building for the dead: events, processes and changing worldviews from the thirty-eighth to the thirty-fourth centuries cal. BC in southern Britain.

Wysocki, M. \& A. Whittle, in prep. On the people of the long barrows: human skeletal remains from West Kennet, Wayland's Smithy and Fussell's Lodge. 
